\title{
Tachyons Particles as a Paradigm for the Existing of the Status of Quantum Metaphysics
}

\author{
Kahsu Abrha Belew \\ Lecturer of Philosophy, Raya University, College of Social Sciences and Humanities, Department of Civics and \\ Ethical Studies, Maichew, Ethiopia
}

\begin{abstract}
As many scientists wrote repeatedly, quantum mechanics is a naturalistic theory and it flourished in 1920 s, so as to solve physical problems through empirical experiment. It is the most vivacious empirical science to explain cosmological puzzles for the last one hundred years consistently. Philosophers of physics evidently argue that the nature of quantum mechanics (sometimes so-called quantum theory or physics) in physics can be associated with the coming of logical positivism (empiricist philosophy) on the philosophy segment. As history of physics showed quantum physics is a one among the many scientific theories which is the most fruitful and applied over. Though scientists were raised intentionally or unintentionally metaphysical questions, but they applied quantum mechanical stance. Accordingly, till $20^{\text {th }} \mathrm{c}$ physicists were simply defending on experiment based reality only (but failed)! However, mid- $20^{\text {th }}$ century later theoretical physicists have changed this experimental physics and it follows revolutionized science. Therefore, there was a paradigm shift from the quantum mechanics stance to quantum metaphysics posture because the revolution in physics is not only to review and reading about the physical world, but also the world of metaphysics too. Thus, in modern societies, a strange and new theoretical quantum science was developed in 1967's by Gerald Feinberg, so called quantum metaphysics which can justify through Tachyon particles so as to realize metaphysical mien. Based on quantum physics postures, Feinberg puts forward an assumption of quantum metaphysics and Tachkyonic particles in general.
\end{abstract}

Keywords: Quantum Physics, Physics, Metaphysics, Quantum Metaphysics, Tachyons, Feinberg

DOI: $10.7176 /$ APTA/82-02

Publication date: January $31^{\text {st }} 2020$

\section{Introduction}

The word 'metaphysics' is the study of the nature of reality in general, asking questions like: what is reality? Is reality some kind of "thing"? Is it one or many? And the like. It limits itself with different puzzles which are exists something beyond the physical world (something that can be proved) and experiment through quantum law. Accordingly, physical scientists applied this experimental science for centuries and it was a fruitful and successful science.

Despite its status as key part of contemporary physics, there is no consensus among physicists and philosophers of physics about the question of what the great empirical success of quantum theory is telling us in relation to the physical world. This has led to a number of philosophical questions known like 'interpretations of quantum mechanics' (Sia, 2017:107).

$\mathrm{Sia}$, added that in "quantum filed the picture is different; there are no quantum states that assign defined values of all physical quantities" (Ibid). so it excludes the totality of metaphysics and this shows quantum field is limited and one-sided.

Accordingly, a modern society has been discovered a new and strange theory so - called quantum metaphysics, and it has been reflected by Tachyons particles which proposed by an American philosopher Gerald Feinberg. Thus, during the time two antagonists philosophy of physics has been emerged: quo quantum mechanics and against quantum mechanics (so-called philosophers of metaphysics). The possibility of particles moving faster than light was first proposed by O.M.P Bilaniuk, V.K. Deshapande, and E.C.G. Sudarshan in 1962.

Although the term they used for it was "meta-particle". In the 1967 paper that coined the term, Gerald Feinberg proposed that Tachkyonic particles could be quanta of a quantum field with imaginary mass. However, it was soon realized that excitations of such imaginary mass fields do not, in fact, propagate faster than light, and instead represent an instability known as Tachyon condensation nevertheless, in modern physics the term "Tachyon" often refers to imaginary mass fields rather than faster than light particles. Such fields have come to play a significant role in modern physics (Feinberg, 1967: 1).

However, the objective of this paper is to show how quantum physics is a science of quantum metaphysics too? In order to achieve this objective, I base my argument on quantum metaphysics in general and Feinberg's concept of Tachyons particles argument in particular. Besides, the paper envisioned as a brief introduction to the quantum metaphysics and Tachyonic particles as well. Finally, the paper is in a position to elucidate the following research questions: how a Tachkyonic particle proves metaphysical disputes? And is quantum mechanics a science of metaphysics too? Finally, by defending Feinberg's argument of Tachyons particles, this paper would show the statuesque of metaphysical assertions and its cognitive justifiability. 


\section{The Philosophy of Quantum Physics ${ }^{1}$}

Before portraying the nature of quantum physics, let's say something about the philosophical view of metaphysics. From the etymological point of view, i.e. the root of the word, metaphysics, means "after the things of nature". It came from two Greek words 'meta' meaning "after", and 'physika' meaning "nature". Metaphysics is thus the study of theory beyond nature which Aristotle called "first philosophy". This now leaves us with the literal or dictionary meaning of metaphysics; however the technical meaning of the word seems like this. Technically, we can say that metaphysics is a branch of philosophy, which studies the nature of reality. This reality refers to the reality of man as a human and non-human world, i.e. God, etc. In this sense, metaphysics translate into the theory of reality (Miller, 1984: 51-54).

Conversely, quantum ${ }^{2}$ mechanics is, obviously, a vital theory of physics filed. For the past several years, all most all scientific theories was seen and calculated by the measurement of quantum mechanics and its analysis and predictions were described through observation and experiment merely. Unlike metaphysics, however, its concerns are much more restricted and it narrows itself into the studying of the natural world alone, where as our experiences of nature may be but one aspect of the total reality that interests philosophers of metaphysics. Besides, quantum physicists are quite interested in the world of nature; they naturally imply primarily the tools of observation and experimentation. Thus, it implies that metaphysical issues were not the business of being practical man who believes by experiment and visible instruments (materialist) for the long period of time (Wong, et.al: 12).

According to American Heritage Dictionary, physical science is defined as "the observation, identification, description, experimental investigation, and theoretical explanation of natural phenomena". The word itself is synonymous with knowledge. Now the vast majority of what we call knowledge has, as its foundation, the information that is provided to us through our five senses. We know where we are and we make conclusions about the world based on the evidence that these five organs of knowledge deliver. The quantum filled channels this knowledge through a discipline known as "The Scientific Method" (Sia, 2017: 106-107).

Accordingly, any philosophy of quantum may consist of the following four steps: 1. Observation and description of a phenomenon or group of phenomena. 2. Formulation of a hypothesis to explain the phenomena. The hypothesis often takes the form of a causal mechanism or a mathematical relation. 3. Use of the hypothesis to predict the existence of other phenomena, or to predict quantitatively the results of new observations. 4 . Performance of experimental tests of the predictions by several independent experimenters and properly performed experiments (Ibid: 107-109).

As a result, the world that we live in today, with its amazing wealth of material goods and the astonishing abundance of knowledge regarding the laws of nature is the direct result of the application of Man"s intelligence to the second question (What is the nature of this vast creation before me?) through the instrument of the scientific method without giving a concern for metaphysical milieu.

Accordingly, I argue that quantum mechanics, scientific theory have been problematic and tricky due to it's an erroneous calculation and one-sidedness. However, now a day severe quantum physicist becomes questioned on the rationality of the quantum mechanics and makes it into the box of skepticism. Hence, different quantum scientists produce a different argument despite their argument would be classified into two major segments: A thinking that quantum mechanics can be proves the world of quantum metaphysics too. This category of thinking can be called pro-quantum metaphysics. Whereas others deny this, call them against quantum metaphysics (or proquantum mechanics).

\subsection{Einstein and Feinberg}

In the last but not the least precedence Albert Einstein has committed a logical error in his special relativity theory. As Bertrand Wong suggested:

Special Relativity stipulates that the velocity of light is constant and nothing can exceed this velocity--- According to Einstein, a faster-than light particle has negative mass/length, which implies that the faster-than-light particle has suddenly changed to the opposite direction in its travel path (which can be interpreted as traveling backwards in time). This seems weird and absurd. Perhaps this prompted Einstein to assert that the velocity of light is the maximum velocity and particle could travel at (2017: 1-2).

Feinberg, an American physicist found Einstein's "mass formula subatomic particle" that faster-than-light and gave a theoretical meaning as Tachyons which are simply hypothetical particles that move faster than the speed of light, according to the theory. And this breaks the law of normal physical or Einstein's theory. Therefore, a Tachyon a subatomic particle moving faster than light according to Feinberg theory. The principal objective of the paper is to show how Tachkynic particles, are hypothetical particles that are considered to be moving more

\footnotetext{
${ }^{1}$ It is also known as quantum physics and quantum theory.

${ }^{2}$ Etymologically, the word quantum is derived from Latin "quatum", meaning "how great" or "how much".
} 
than the speed of light, so there is a higher speed of light. However, many physicists were siding with the box of Einstein's theory of relativity (but fail)! Their reason is with the assumption of relativity is the foundation of modern physics along with the quantum theory. Their reason seems to be unscientific or fallacious one.

Over a century ago, Albert Einstein has anticipated odds things happening on spaceship travelling at speeds close to that of light (roughly $300,000 \mathrm{~km} / \mathrm{s}$ ) and impossible things happening when travelling at more than the speed of light (Ibid: 2-4). It is not necessary here and now to go into why this should be, but these predictions are enough to convince much smarter people than me that the speed of light is affixed, the fundamental speed limit in the universe and that no material objects can ever attain or exceed this crucial speed. However, some theoretical physicists have gone for a walk on the wild side by speculating on the possible existence of particles of matter which always travel faster than light, avoiding the complications of acceleration past the cosmic speed limit (Wong, 2017: 2)

Physicist then just applied laws of special relativity to check the behavior of articles when they exceed the speed of light and it turned out that the mass of the Tachyon is an imaginary number! ("Numbers which involve iota or square root of -1 "). In order to bring them down to the speed of light, they would require an infinite amount of energy, just the opposite case for "Tardyons". Surprisingly enough, theoretically the total energy of Tachyons increases as they go at even faster speeds and becomes zero as they approach infinite speed. Although the theories of tachyons were developed first in 1980's and active research is still going on them, we have a long way to go before we might actually find one (Feinberg, 1967: 1089).

\section{Beyond Quantum Physics}

As many scientists wrote repeatedly, quantum mechanics is a naturalistic theory and it flourished in 1920 s to solve physical problems through empirical experiment. It is the most empirical science to argue nature for the last one hundred years consistently. That is why quantum mechanics is fruitful and victorious science. The nature of quantum mechanics in physics was associated with the coming of logical positivism (empiricist philosophy) ${ }^{1}$ on the philosophy segment. Currently, metaphysical questions tend to the questions of quantum mechanics too. Scientists raised directly or indirectly metaphysical questions and problems, and then they are trying to solve them by quantum mechanical stance.

Now a day, there is a paradigm shift from the quantum mechanics approach to quantum metaphysics status, the revolution in physics is not only to review and rearguing about the physical world, but also the world of metaphysics too. Regarding this, as Nicholas Ruddick mentioned, Huxley discusses that:

...At once to affirm that he had no doubt that evolution was an amoral cosmic process to which all life on earth, including mankind, was subject, and to propose that human beings had an innate ethical sense which they had the responsibility to attempt to apply in their dealings in the world: 'social progress means a checking of the cosmic process at every step and the substitution for it of another, which may be called the ethical process' (2000: 119).

According to Fill North, traditionally physicists endorsed that the law of physics is intended only to understand and analyze the physical world, and it is guided by "... three dimensions of ordinary space (or four dimensions of ordinary space-time...)" (2013:1). This implies that, the three-dimensional space is the one that precisely correspond to the world's fundamental physical space. As a result, physicists used for scientific philosophizing, and space and time are crucial to analyze it. Any scientific theory can detect any empirical questions and after all it's possible to achieve the cosmological truth through space-time dimensional law.

Thus, the dynamical laws are escorted to the vital nature of a world. Before discussing the fundamental nature of "tachyons", let's illustrate "dark energy" and "dark matter". At this point of view, Marlene Gotz notes: "Dark energy is a hypothetical form of matter. It has to be postulated to describe the phenomenon which could not be explained by known forms of matter ... such a particle does not exist within the standard model of particle physics".

The philosophical claim of this passage is that there is one thing that cannot be seen and removed from the form of normal energy of quantum mechanics formula that is dark energy or sometimes called "tachyons". As defined above, tachyons are simply particles that move higher than the speed of light that can be unseen through the normal dynamical law and help to demonstrate non-visible entities, like metaphysics. Moreover, as Michael Soberepera claimed in his paper entitled Tachyons and Other Things Fast: A Study of Superluminal Motion, tachyons are a hypothetical particle that cannot be guided by the laws of normal physics (space time dynamical law) but "which has never been observed".

What a fascinating point in his article Dark matter, Dark Energy and Gravitational Properties of Antimatter, Hajudukovic argues, "... the current concordance model of cosmology, the Earth, the stars, the galaxies and everything we are familiar with, make up less than $5 \%$ of the total matter and energy in the universe. The rest of

\footnotetext{
${ }^{1}$ In the early $20^{\text {th }} \mathrm{C}$, the school of philosophy known as logical positivism arouses, in a "Vienna Circle"1. The school of philosophy with a kind of radical empiricism center piece with the principle of verification. According to the principle of verification metaphysical statement has a meaning if and only if either "analytically true or empirically verifiable". It implies that, to be a meaningful science if it fulfilled the above two requirements.
} 
$95 \%$ is dark energy ..." (Hajdukovic, $)^{1}$. Accordingly, the unobservable form of the world or dark energy contains $95 \%$ of the total energy, from the known or observable universe. And dark matter contains 5\% of the total world. This implies, for a several years human beings have acquired 5\% knowledge of the visible world merely, but they have missed the largest and biggest world known as metaphysical world/dark energy or $95 \%$.

Accordingly, the concern is with the largest and biggest word even it was abandoned one called metaphysical world (95\%). As Plato putted, metaphysics or reality is classified into two: (I) "reality of the physical objects in space and time, which are objects of the senses (II) The reality of concepts, ideas, forms, or essences which are objects of thought" (Lavine, 1984: 26). However, the paper is going to examine the second world, the world of metaphysics. It lodges itself with some fundamental questions which are beyond physical experience or beyond our senses.

Thus, tachyons particles are useful so as to validate metaphysical world. This invisible world can be visible by the strange and new particle so called" tachyons". In this context, metaphysical assertions can be determined and proved through tachyons particles. The main question here is if we have applied dark matter in order to elucidate the visible universe through dynamical law, then how is it possible to hold non-visible world? As mentioned above, dark energy is not just like dark matter, describing the observable world merely, but it is something beyond the observable world. This can be known by Tachyons and helps us to reveal the unknown world (dark energy or metaphysics). Dark matter is nothing rather than a collection of particles that are not capable of "absorb", "reflect", or "emit light. Moreover, it is something a material and that can be seen, observed through dynamical law. Regarding this, Marco Baldi states that, "dark energy and dark matter are two components might directly interact with each other...(2009: 23).

Let's turn to the chief objective of the paper that can be defined as a hypothetical particle called that goes beyond the speed of light and helps to analyze the unexplained issues such as metaphysics, ethics, mysterious and abstraction events. In this regard, Allori notes that "all quantum theories should be regarded as theories in which physical objects are constituted by a primitive ontology. The primitive ontology is mathematically represented in the theory by a mathematical entity in three dimensional space, or space time" (N.d: 5).

For centuries, physicists were simply permitted within the box of Albert Einstein's philosophy of physics, which approaching with three space and one time dimension. These also give services to them in order to realize physical world by neglecting the larger world, despite currently it is a fallacious logical reasoning of special theory of relativity. In contrast, philosophers of physics or metaphysicians going to be denied the dogma of space and time reconciliation philosophical investigation. The reason is that the physical world or human knowledge of the visible cosmos contained 5\%, it follows that we have been known only 5\% about the physical world. This implies that, they rejected the larger world which contained 95\%; that is, the world of metaphysics (Hajdukovic,).

Besides this, North states that the world is built-up, in accordance to its dynamical law. Because, as he argued physics is principally functional when questions needs to answer; about how and why physical objects move around and interact with one another, it follows that the "dynamical laws are generalizations describing this behavior". Therefore, the means to know is if and only if appropriate with what Einstein's told us /did or with dynamical laws so such kind of epistemology is a matter of interrelating space-time, meaning three space and one time. As I mentioned above, this dynamical law is a so-called space-time dimension or structure of the physical world (North, 2013: 3-4).

The immediate question here is that, is it possible to determine metaphysical (non-physical) utterances world through dynamical laws? Optimistically, I say yes, it is possible, but a method for physics is not a method for metaphysics too, due to their methodological approach to discover something reality becomes different. It is to mean that the dynamical law of the physical world is not the law of metaphysical world too. When dynamical law is for physics, Tachyon particle is for metaphysics. From history of physics, for instance, take Newtonian physics, dynamical laws is not only determined to show "... the space-time structure, the dynamical laws tell us that, fundamentally, there are particles, which travel along straight paths unless acted on by a net external force..." (North, 2013: 6). In this context, particles are referring to something that does not determine the dynamical law, but something higher dimension called Tachyon, a particle that moves more than the speed of light.

The alarm of the paper is to show the relevance of quantum theory of Tachyons in relation to metaphysics. Quantum mechanics is, therefore, not merely useful for 5\% (physical world) but also 95\% (world of metaphysics) too. Hence, it is possible to realize or recognize metaphysics through quantum mechanics. This can be considered as a paradigm shift from binding on quantum physics to metaphysics. As quantum mechanics of metaphysics contend there is one higher dimension that exists beyond the showed world, and it contains the largest percent $(95 \%)$ or metaphysics. This paper shall defend this depending on the quantum mechanics of metaphysics using Feinberg's idea of Tachkyonic particles, below. Having this in our mind, now let's turn to the principal objective of the paper that is how quantum mechanics served for metaphysics through the Feinberg's argument of Tachyons particles?

\footnotetext{
${ }^{1}$ The $95 \%$ and 5\% can be calculated by Lamda-CDM model (it is some sort of standard model of cosmology).
} 


\section{Feinberg's Concept of Tachyonic Particles and Metaphysical Status}

Nowadays, all eyes of physicists come to think about a new and strange quantum field, known as Tachyon. Gerald Feinberg is a one among the prominent figure of quantum mechanics and the well-known philosopher of physics that has developed a strange but an actual idea of quantum mechanics in physics by deconstructing experiment based quantum filed and the view that traveling more than the speed of light is not exist even unthinkable. Etymologically, the word Tachyons derived from Greek "tachy", meaning "rapid", it was coined in 1967 by Gerald Feinberg. In Einstein's well-known discourse was, it is often nothing can travel faster than the speed of light (https: www. Scientific American.com).

However, Feinberg's main concern is with the philosophical question, are hypothetical particles that move faster than light? As discussed above, such particles were first alleged by Gerald Feinberg in 1967 and he argued in his paper entitled "Possibility of Faster-Than-Light Particles" as follows:

We consider the possibility of describing with the special theory of relativity, particles in space like four-momentum, which therefore have velocities greater than that of light in vacuum. The usual objections to such particles are discussed, and they are found to be unconvincing within the framework of relativistic quantum theory. A quantum field theory of noninteracting, spineless, faster-than-light particles is described" (1967: 1089).

Moreover, he believes that:

The field theory is Lorentz-invariant, but must be quantized with Fermi statistics. The associated particle theory has the property that the particle number is not Lorentz-invariant, and the noparticle state is not Lorentz-invariant either. Nevertheless, the principle of relativity is satisfied. The Lorentz invariance implies relation between emission and absorption processes, in contradiction to the usual case. Some comments are made about the problem of introducing interactions into the field theory (Ibid).

The passage clearly shows that although most physicists believe that faster than the speed of light cannot exist because not consisting with the laws of physics and Einstein's hasn't ordered to recognize as an actual physics if such metaphysical particle proposed. Besides this, despite if such particles did exist, then particle in alarmingly leads to violations of causality. Therefore, this kind of notion tends to contradict with Einstein's theory of relativity. As we all know Einstein argued that "the speed of light absolute the same for all observers, whatever their speed". This saying is not open to encounter arguments like there is a hypothetical particle that travel more than the speed of light so-called Tachyon, is a field within imaginary mass, although Tachkyonic particle is a purely hypothetical concept that violated Einstein's special relativity theory (Wong, 2017: 1-2).

However, in contrast, various philosophers of physics assume that it can never be exist faster than the speed of light due to the following reasons:

I. It contradicts with the law of actual physics

II. No consistent with the recognized dynamical law of quantum physics

III. No one experimental justification to show the existence of tachyons, no one can experimentally prove the existence of such particles yet. Or no possibility to say Tackyonic particles are moving higher than light because it is not approached to sense perception (Ibid, 2-3, Sia, 2017: 110-13).

Paradoxically, possibilities might be occurred and thinks that particles moving faster than light. Pro this argument such as: O.M.P. Bilaniuk, U.K. Deshpande and Gerald Feinberg. Generally, the nature of Tachyons particles is nothing but particles that can go faster than the speed of light. When experimental scientists heard this, they will be surprised. Their sole reason is that traditionally called as a father of modern physics, Albert Einstein, didn't documented that nothing can go faster than the speed of light, nothing is beyond the speed of light or doesn't exist if it is occurring. But, he sort of did, but there's a slight ambiguity. And ambiguity is Einstein's speed relativity essentially displays that nothing can twitch out slower than the speed of light, then speed up and cross the speed -of-light fence into the faster than the speed-of-light realm.

However, that, at least, opens the possibility that maybe there's a particle that always and forever is travelling faster than the speed of light so it never needed to cross over the fence. As connoted above, such particle is called a Tachyon. Now most of us don't think that Tachyons are real. If they are real, they give rise to same strange phenomena. For instance, if I had a Tachyon gun and fired it at you, then, according to same people watching it, they would say the Tachyon hit your body before pulled the trigger. Tachyons cause destruction on philosophies of "causality". So for that reason, various experimental scientists don't contemplate that these things are actual and factual. But, Feinberg has shown by philosophizing Tachyons particles.

Moreover, in his article entitled" possibility of faster- than-light particles", states that Tachyons are hypothetical particles and can move faster than the speed of light. And the word "Tachyon" was devised by him, in 1967. He tries to understand quantum metaphysics by such particles. Therefore, he has shown a new field of quantum (quantum metaphysics) and particles (tachyons) for those who have an ambition to be a theoretical physicist believe that everything can be solved and calculated quantum mechanics law-meaning space-time dynamical law. Fienberg strongly, believe that a Tachyons particle has a condition to justify metaphysical issues 
such as: the existence of God, Satan and so forth. Based on Tachyons metaphysical problems can be solved. Hence, quantum mechanics served for physical problems, but quantum metaphysics for nonphysical problems (Feinberg, 1967: 1098).

Now let's depict the argument how Tachkyonic particles disrupt Einstein's standard of causality. Obviously, causality is a fundamental element of physics in general and Einstein's quantum mechanics in particular. If tachyons can convey information faster than light, then rendering to Einstein's relativity, they disrupt causality, leading to sound inconsistencies of the "kill your own grand faster" type.

This is frequently exemplified with thought experiments such as "tachyons telephone paradox" or "logically pernicious self-inhibitor". The tricks can be comprehended in terms of the relativity of simultaneity in special relativity, which utters that diverse "inertial" orientation settings will fluster on whether the two actions at diverse circumstances occurred "at the same time" or not, and they can also fluster on the order of the two actions. If one of the two actions presents the sending of a signal from one location and the serial actions signifies the assumption of the similar signal at another circumstance, "then as long as the signal is moving at the speed of light or slower the mathematics of simultaneity ensures that all reference frames agree that the trains missive-event happened before the perception -event however, in the case of a hypothetical signal moving faster than light" (Ibid, 1089, Sia, 2017: 110-11).

Generally speaking, Feinberg coined/proposed Tachyons particles so as to prove metaphysical utterances cognitively though numerous empirical physicists have rejected his theory, because they assume that nothing is faster than light, even if it exist it cannot follow the law of physics. In relation to this, my concern with Tachyons philosophical assumptions; simply particles that move more than the speed of light; it is not guided and measured by a dynamical law (three space and one time dimension). However, it is guided and measured by a "reasonable guide" and it investigates metaphysics. Furthermore, this principle is employed to the fundamental level; it says that, to infer just that fundamental structure and ontology (North, 2013: 7).

Furthermore, in quantum mechanics, to devise the dynamics, we merely rely on three space and one time dimension. In contrast, Tachyons are inseparable or "cannot be broken down into individual three Tachyons". So, the fundamental ontology maintains Tachyons then exists in it. I support Feinberg and North's argument that Tachyons presupposed by question mechanics, so we are going to formulate the basic nature of metaphysics in our mind governed and guided by this higher dimension (Tachyons). Thus, the preliminary function of Tachyon particles is to provide an impression, conviction, emotion and feeling and then we gained an impression/ feeling or they are an extra dimension which is encoded but we taste them. If we have fallen on something, then we lead to evaluate for it. For instance, we can evaluate the cognitive nature of metaphysical statements as true or false through Tachyons. Because Tachyon gives similar information to our brain and finally we feel similarly (Amaha, 2017).

In this context, Hilbert was coined with "high-dimensional such ontology" this is called "Hilbert space, is best interpreted as an abstract states pace" (North, 2013: 11). He rejected the space-time dimension because he argues that there is a higher dimension that exists beyond Physics. This higher dimension is, as we all know Tachyons, things that are not able to guide by a dynamical law. This is my central argument that I want to pinpoint; besides, I base my argument on Hilbert's conception of quantum mechanics of metaphysics that is every metaphysical assertions are cognitively justifiable. In this regard, since quantum world is formulated by space and time dimensional law. Obviously, quantum mechanics can be able to justify through three spaces and one time dimensional law. In contrast, this; this dimensional law is not applicable for quantum ontology. Because, as Feinberg's argued Tachyons are exists in our inner body, especially in our faith. The occurrence of Tachyons denotes that how metaphysical and mysterious statements can be proved (Ibid).

Accordingly, metaphysical assumptions, statements cannot be grasped through three space dimensions. To mean that being a table should be measured through three space dimensions; the table exists in three spaces. So, this has nothing to do with metaphysical issues like God's existence, because this is not the simplest one to prove empirically through dynamical law. We do not wonder why the dynamical law is relevant to metaphysics. We do have Tachyons particles to solve such puzzles.

According to Hari, things that we have seen, tasted, or generally proved and grasped through our five senses are called quantum mechanics or it bases on the sense perception. Simply it can call "three spaces and one time dimension". Three space dimensions mean that: height, width and depth. However, if we add time on it, then it is possible to call one time dimension. So, what we know something here is that, in our day-to-day experience, we practiced it and we live our life with such normal process.

My interest here is that, like Hilbert and Feinberg, I also believe that there is another higher dimension or there is one dimension that exists beyond space and time dimension. And this higher dimension is justified, known, and proved through Tachyon particles. Besides, like Hilbert I certainly argue that, if we blend the two components (physical and non-physical or physics and metaphysics), finally we found something perfect, for instance, metaphysics perfection. Besides, perfection might be cognitive or metaphysics. In this regard, Marco Baldi argues, "... that these two components might directly interact with each other... (2009: 23) and we found something 
perfect that perfection is called metaphysics. Mean that metaphysical assertions are capable of being true and false, justifiable, and cognitively meaningful.

As it has illustrated above, the content of the universe that I can see the physical world by using my senses is merely 5\%. This implies that we can know the universe only $5 \%$. What about $95 \%$ ? They remained $95 \%$ is metaphysics. That is why I am interested in this large world. So, Metaphysicians believe that if we exactly blend $95 \%$ and $5 \%$, then we found something cognitive, the cognitive of metaphysics.

As it has been mentioned earlier, there are two significant points: "photons ${ }^{1}$ and Tachyons". We used photons for signals, like computer, mobile. Unlike photons, we used Tachyons in metaphysics because their speed is higher than photons. Simply Tachyons can give us an impression, similar feelings and influenced us to have something like metaphysics cognitively. In general Tachyons seen by "endocrine gland" and gives similar feelings and they exist in our fat. Endocrine gland is like a chain of amino acid and gives the senses of metaphysics, even before we have borne. Moreover, the main function of Tachyons is to give us information for all humans and feeling, emotion, attitude, and thinking. In short, they give us similar feeling, emotion, attitude, and so forth. Hence, if the feeling is entered through Tachyons, then we start to think and evaluate it. Such evaluation might metaphysical. This is deduced that both metaphysical can be justified.

Let's make clear the point based on the following analogical argument: for instance, accurately one bird can fly, it cannot fall down, because her ability is based on "instinct" but she can never think, evaluate and recognize what she has done. The only reason is that she does not have enough brain, she merely flying by instinct. I argue that, since the endocrine gland or Tachyons are something which helps us in order to have metaphysical understanding. So, cognitively we can know metaphysical scenarios judgments, even if through Tachyons we knew abstraction, mystery and metaphysics.

\section{Conclusion}

Of course, I have not a full confidence to multiply Einstein's total theory by zero. However, Feinberg has been deconstructed this and he argued that there is an article that always moves faster than the speed of light. This article is so called Tachyon or Tachkyonic particles. Hence, Tachyon is a particle that moves faster than the speed of light. Therefore, Feinberg coined the term tachyon; it breaks down the unthinking of thinking that confined by severing physicist. This paper investigated the validity of Feinberg's notion of Tachkyonic particles and aimed that by using Tachyons it can be possible every metaphysical assertions like what quantum mechanics done. The only difference is that the way of application. According to quantum theory, any problems and challenges can be solved and proved through observation and experiment no need further experiment like a thought experiment, in contrast to quantum metaphysics, something can solve and checked up through Tachkyonic particles, although difficult to make an analysis based on experiment, rather they used though experiment.

In 1967 Feinberg that coined the term, and proposed Tachkyonic particles could be the part of quantum field. Tachyon or Tachkyonic particle is a hypothetical particle that always moves faster -than-light. Nevertheless, most physicists believe that faster-than-light particles cannot exist, because they are not consistent with the known laws of physics. If such particles did exist, they could be used to build a Tachkyonic particle anti telephone and send signals faster than light, which would lead to violations of causality.

\section{References}

Allori, Valia. (N.d). On the Metaphysics of Quantum Mechanics. Available at: vallori@niu.edu.

Baldi, Marco. (2009). Interactions between Dark Energy and Dark Matter. Dissertation of the Faculty of Physics, At the Ludwing Maximilan University Manic.

Gotz, Marlene n.d, Proceedings Astronomy from 4 Perspectives.

Hajdukovic, S. Dragan. (N.d). Dark Matter, Dark Energy and Gravitational Properties of Antimatter. dragan.hajdukovic@cern.ch.

Hari, D. Syamala. (2011). Mind as Tachyons: How Tachyon Changes Quantum Potential and Brain Creates Mind... Miller, ED. L. (1984). Questions that matter. 6thed, university of Colorado

Miller, L.ED. (1984). Questions That Matter: An Invitation to Philosophy. McGraw- Hill Book Company.

North, Fill. (2013). The Structure of a Quantum World. Oxford University Press.

Website: (https: www. Scientific American.com) article /what-is-known-about-tachy?).

Sia, D. Paolo. (2017). Quantum Physics, Metaphysics, Theism: Interpretations, Ontologies, Theological Remarks. University of Bolzano

Sia, Paolo, Di. (2017). Quantum Physics, Metaphysics, Theism: Interpretations, Ontologies, Theological Remarks: University of Bolzano: Available online at www.worldscient ificnews.com

Wilberg, P. Acharya. (2009). The Metaphysics of 'Dark Matter'. The Foundation of Physical Science.

\footnotetext{
1 “G.N. Lewis, in 1926, coined the word 'photon' to describe something different from the Einstein light quantum (Lamb, W.E. (1995). AntiPhoton. Optical Sciences Center, University of Arizona). And photons are simply particles or signals used for dark matter or sensible world, such as radio, TV, computer and so forth.
} 
Wong, Bertrand, Eurotech, Branch, S'pore. (2017). Detection of Tachyons. Available at: http://gsjournal.net/Science-Journals/Research\%20Papers-Unification\%20Theories/Download/6927 